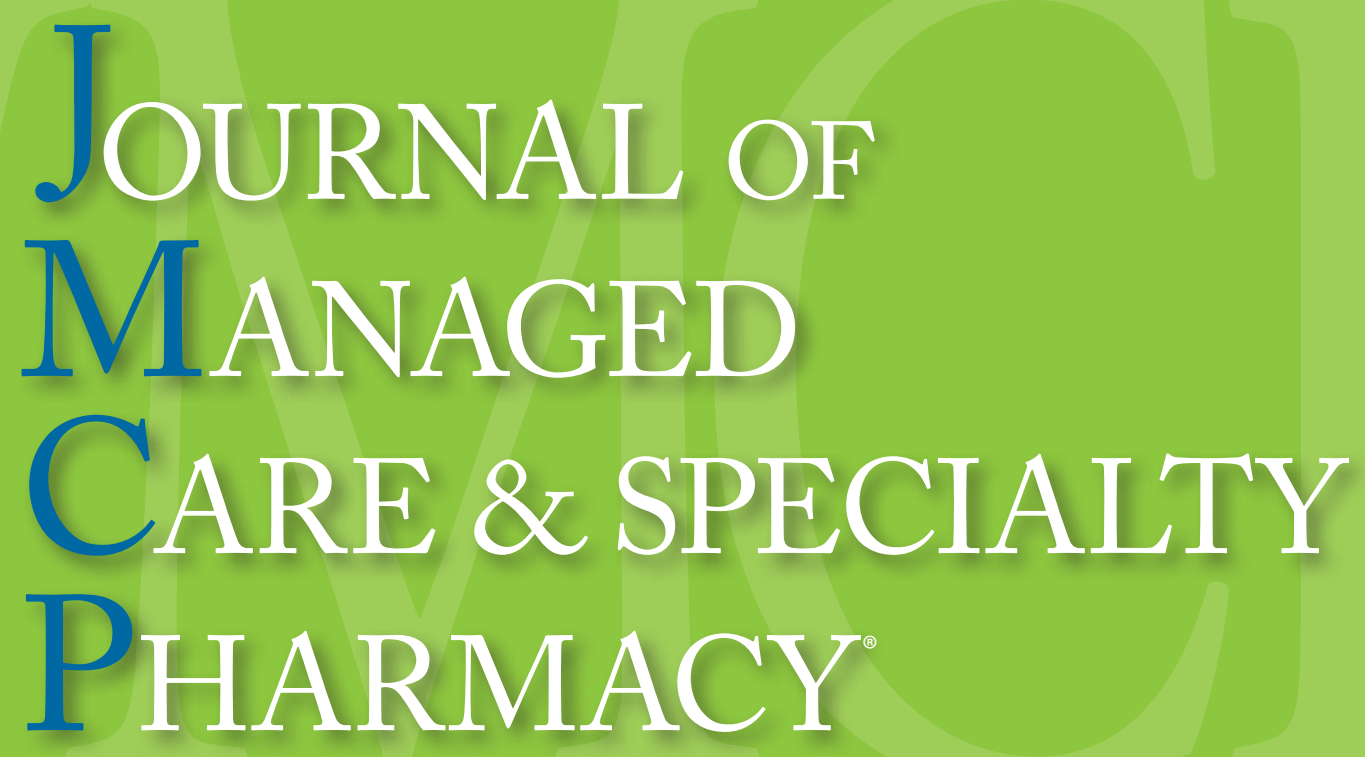

Supplement $\square$ Volume 25 — Number 3-b - March 2019

\title{
Evaluation of Treatment Patterns and Costs in Patients with Prostate Cancer and Bone Metastases
}

Lonnie Wen, RPh, PhD; Jianying Yao, MS; and Adriana Valderrama, PhD, MBA 


\section{J

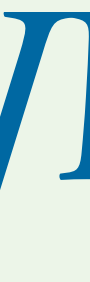

Publisher

Susan A. Cantrell, RPh, CAE

Chief Executive Officer

Academy of Managed Care Pharmacy

\section{Editor-in-Chief}

Laura E. Happe, PharmD, MPH

727.488.2700, lhappe@jmcp.org

\section{Assistant Editors}

Donald G. Klepser, PhD, MBA

University of Nebraska Medical Center, Omaha, NE

Melissa S. McCart, PharmD, MS

Xcenda, Palm Harbor, FL

Karen L. Rascati, PhD

University of Texas College of Pharmacy, Austin, TX

\section{Managing Editor}

Jennifer A. Booker

703.317.0725,jmcpreview@amcp.org

\section{Production Editor}

Carol Blumentritt

602.616.7249, cblumentritt@amcp.org

\section{Graphic Designer}

Margie C. Hunter

703.297.9319,mhunter@amcp.org

\section{Account Manager}

Bryan Bonder

American Medical Communications

610.715.4384,Bbonder@americanmedicalcomm.com

Maria Sercia

American Medical Communications

908.216.1548,MSercia@americanmedicalcomm.com

This supplement to the Journal of Managed Care \& Specialty Pharmacy (ISSN 1944-706X) is a publication of the Academy of Managed Care Pharmacy, 675 North Washington Street, Suite 220, Alexandria, VA 22314; 703.684.2600; 703.684 .2651 (fax).

Copyright $(\odot)$ 2019, Academy of Managed Care Pharmacy.

All rights reserved. No part of this publication may be reproduced or transmitted in any form or by any means, electronic or mechanical, with out written permission from the Academy of Managed Care Pharmacy.

POSTMASTER: Send address changes to JMCP,

675 North Washington Street, Suite 220, Alexandria, VA 22314

\section{Supplement Policy Statement}

Standards for Supplements to the

Journal of Managed Care \& Specialty Pharmacy

Supplements to the Journal of Managed Care \& Specialty Pharmacy (JMCP) are intended to support medical education and research in areas of clinical practice, health care quality improvement, or efficient administration and delivery of health benefits. The following standards are applied to all JMCP supplements to ensure quality and assist readers in evaluating potential bias and determining alternate explanations for findings and results.

1. Disclose the principal sources of funding in a manner that permits easy recognition by the reader.

2. Disclose the existence of all potential conflicts of interest among supplement contributors, including financial or personal bias. 3. Describe all drugs by generic name unless the use of the brand name is necessary to reduce the opportunity for confusion among readers.

4. Identify any off-label (unapproved) use by drug name and specific off-label indication.

5. Strive to report subjects of current interest to managed care pharmacists and other managed care professionals.

6. Seek and publish content that does not duplicate content in the Journal of Managed Care \& Specialty Pharmacy.

7. Subject all supplements to expert peer review.

\section{AUTHORS}

Lonnie Wen, $\mathrm{RPh}, \mathrm{PhD}$, is Deputy Director at Bayer Pharmaceuticals with the U.S. Field Data Generation and Observational Studies team. In this role, Wen is responsible for leading the development and implementation of U.S. Data Generation and Observational Studies research in the field. In addition, she leads the dissemination and communication of health economics and outcomes data for health plans, integrated delivery networks, and other health care systems such as the Department of Veterans Affairs Medical Centers. Wen has a bachelor of science degree in pharmacy, a master of science degree, and a doctorate in pharmacy administration from the University of Texas at Austin. She completed a postdoctoral fellowship in health services research at the Veterans Evidence-Based Research Dissemination and Implementation Center at the South Texas Veterans Health Care System in San Antonio. She currently resides in Jackson, Mississippi.

Jianying Yao, MS, is an Associate Director at Bayer Pharmaceuticals with the Global Data Analytics Group in Data Generation and Observational Studies team. Yao is a senior biostatistician, with $20+$ years of extensive experience in designing and conducting health outcomes research. She is seasoned in advanced quantitative and analytical skills over large relational databases, with strong expertise in integrated medical and pharmacy claims data analytics. Yao holds a master of science degree in biostatistics from the University of Illinois at Chicago and a bachelor of science degree in physics from the Shanghai Second Institute of Education.

Adriana Valderrama, PhD, MBA, is a Director in the Center for Observational and Real-World Evidence (CORE) at Merck. Previously, Valderrama accumulated more than 10 years of U.S. and global experience in Health Economics and Outcomes Research (HEOR) at Ipsen Pharmaceutical and Bayer Healthcare. She was responsible for oncology in the U.S. at Bayer Healthcare and was the lead for multiple oncologic agent launches in the US and global HEOR department at Bayer, supporting several countries in their health technology assessment submissions. Valderrama holds a doctorate in pharmacy administration and health economics and a master of business administration degree. 


\section{Table of Contents}

\section{Evaluation of Treatment Patterns and Costs in Patients with Prostate Cancer and Bone Metastases \\ Lonnie Wen, RPh, PhD; Jianying Yao, MS; and Adriana Valderrama, PhD, MBA}

\section{S2 Abstract \\ S3 Methods \\ S4 Results \\ S7 Discussion \\ S9 Conclusions \\ S10 References \\ S11 Appendices}

\section{Disclosures}

Funding for this study was provided by Bayer HealthCare Pharmaceuticals. All authors were employees at Bayer HealthCare Pharmaceuticals at the time this study was conducted. 


\title{
Evaluation of Treatment Patterns and Costs in Patients with Prostate Cancer and Bone Metastases
}

\author{
Lonnie Wen, RPh, PhD; Jianying Yao, MS; and Adriana Valderrama, PhD, MBA
}

\begin{abstract}
BACKGROUND: There are a lack of guideline recommendations for patients with metastatic castration-resistant prostate cancer (mCRPC) undergoing treatment progression and sequencing. Understanding treatment patterns and associated utilization and costs may help inform stakeholders and guide decision making.
\end{abstract}

OBJECTIVE: To describe treatment patterns and health care costs in prostate cancer $(\mathrm{PC})$ patients with bone metastases treated with agents approved by the FDA for mCRPC.

METHODS: 2 large integrated claims databases (MarketScan and PharMetrics) were used to identify males aged $\geq 18$ years who were diagnosed and treated for PC (ICD-9-CM code 185.xx or 233.4) with bone metastases (ICD-9-CM code 198.5) from June 2013 to September 2014. Patients were required to be continuously enrolled for $\geq 6$ months before and after initiation of treatment with abiraterone, cabazitaxel, docetaxel, enzalutamide, mitoxantrone, radium-223, sipuleucel-T, or other chemotherapy. Study endpoints included lines of therapy, health care resource utilization per patient per month (PPPM), PPPM costs, and mortality rate. Descriptive analysis was completed for the study sample, and survival function was calculated via Kaplan-Meier estimates.

RESULTS: There were 953 patients meeting all inclusion criteria in the MarketScan database and 565 patients in the PharMetrics database. The median follow-up time was 18 months (interquartile range [IQR] =14-23) for MarketScan and 14 months (IQR =11-18) for PharMetrics. Mean age (SD) was $71( \pm 10.7)$ and $66( \pm 9.3)$ years, respectively. Before mCRPC treatment initiation, patients received palliative radiation therapy and bone antiresorptive therapy. For MarketScan and PharMetrics, respectively, $14.0 \%$ and $18.2 \%$ of patients received radiation therapy, $36.1 \%$ and $40.0 \%$ received denosumab; $16.5 \%$ and $16.8 \%$ received zoledronic acid; and $0.2 \%$ and $0.8 \%$ received pamidronate. Across both databases, abiraterone was the most commonly received bone metastasis treatment agent across all lines of therapy, except fourth line. Radium-223, cabazitaxel, and mitoxantrone were the least utilized therapies. The median cost PPPM during the post-index period was $\$ 10,916$ (IQR $=\$ 5,334-\$ 13,457$ ) in MarketScan and $\$ 10,292$ (IQR $=\$ 7,245-\$ 14,699)$ in PharMetrics. The cost PPPM during the 6-month pre-index period was $\$ 2,643$ (IQR $=\$ 850-\$ 4,357)$ in MarketScan and $\$ 2,742$ (IQR $=\$ 1,484-\$ 4,730)$ in PharMetrics.

CONCLUSIONS: Patients were treated mainly with abiraterone across most lines of care, with radium-223, cabazitaxel, and mitoxantrone as the least utilized therapies. Median costs PPPM increased by approximately $\$ 8,900$ after initiation of FDA-approved agents for mCRPC, with the largest increase in cost stemming from oral medications.

J Manag Care Spec Pharm. 2019;25(3-b):S2-S11

Copyright @ 2019, Academy of Managed Care Pharmacy. All rights reserved.

I ne United States, prostate cancer (PC) is the most common noncutaneous malignancy among men and the third-leading cause of cancer-related death in males. It is estimated that there were 164,690 new cases of PC and 29,430 deaths in 2018. ${ }^{1}$ Of men diagnosed with PC, approximately 6 in 10 cases are in men aged 65 years or over. The median age at the time of PC diagnosis is 66 years, with rates of death highest among men aged $75-84$ years. ${ }^{1}$

Approximately 30\% who are diagnosed early may experience disease progression to metastatic PC despite receiving previous curative treatment with a prostatectomy or radiation therapy. ${ }^{1,2}$ Patients with metastatic PC are typically treated by reducing levels of androgens in the body. Androgen deprivation can be achieved reversibly through medical castration (i.e., hormonal therapy, also known as androgen deprivation therapy [ADT]) or irreversibly through surgical castration. ${ }^{3}$ Despite these treatments, $10 \%-20 \%$ of all PC patients progress to castration-resistant prostate cancer (CRPC) within 5 years of follow-up. ${ }^{4}$ Androgen-independent phenotypes tend to emerge subsequently, with median overall survival (OS) of 23-37 months from the start of ADT. ${ }^{5}$ Disease progression in CRPC may present as a continuous rise in prostate-specific antigen, progression of pre-existing disease, or appearance of new metastases. Of those diagnosed with CRPC, 84\% present with metastases at the time of diagnosis, also known as metastatic castration-resistant prostate cancer (mCRPC). ${ }^{6}$ The incidence of mCRPC in the United States was estimated to be 36,100 cases in 2009 and is projected to increase to 42,970 cases by 2020 . $^{7}$

When PC progresses from localized or regional disease to metastatic disease, the 5-year relative survival rate drops from nearly $100 \%$ down to $29.3 \% .^{2}$ In mCRPC patients, $80 \%-90 \%$ present with bone metastases, which significantly lower the 5 -year survival rate to $3 \%$ versus $56 \%$ in patients without bone metastases. $^{8-12}$

The presence of bone metastases has been strongly linked to increased resource utilization and costs. ${ }^{13,14}$ In comparison with PC patients without bone metastases, those with bone metastases have increased use of skilled nursing facilities $(22.3 \%$ vs. $8.1 \%$ ), hospice (20.0\% vs. $4.8 \%$ ), and hospitalization $(60.9 \%$ vs. 43.1\%). ${ }^{13}$ Moreover, these patients have longer hospital stays (mean of 3 days longer). In fact, overall hospitalization costs of PC patients with bone metastases versus without differed by more than $\$ 2,000$ per visit between 2006 and 2010. ${ }^{15}$

There are many agents available for treatment of mCRPC with demonstrated survival advantages. Guidelines from the National Comprehensive Cancer Network (NCCN) and American Urological Association (AUA) recommend drugs that target the androgen axis (enzalutamide and abiraterone), immunotherapy (sipuleucel-T), and chemotherapy (cabazitaxel, docetaxel, and mitoxantrone). Targeted alpha therapy agents, a form of targeted radiation, such as radionuclide (radium-223 dichloride) have shown increased survival and decreased time to symptomatic skeletal events, defined as first use of 


\begin{tabular}{|c|c|c|c|c|}
\hline & \multicolumn{2}{|c|}{ MarketScan } & \multicolumn{2}{|c|}{ PharMetrics } \\
\hline & $\mathrm{n}$ & $\%$ & $\mathrm{n}$ & $\%$ \\
\hline Patients & 953 & 100.0 & 565 & 100.0 \\
\hline \multicolumn{5}{|l|}{ Age, years } \\
\hline $35-54$ & 51 & 5.4 & 53 & 9.4 \\
\hline $55-64$ & 266 & 27.9 & 239 & 42.3 \\
\hline $65-79$ & 384 & 40.3 & 207 & 36.6 \\
\hline$\geq 80$ & 252 & 26.4 & 66 & 11.7 \\
\hline Mean age (SD) & $71(10.7)$ & - & $66(9.3)$ & - \\
\hline \multicolumn{5}{|l|}{ Region } \\
\hline Northeast & 231 & 24.2 & 163 & 28.8 \\
\hline North Central/Midwest & 275 & 28.9 & 195 & 34.5 \\
\hline South & 291 & 30.5 & 163 & 28.8 \\
\hline West & 141 & 14.8 & 44 & 7.8 \\
\hline Unknown & 15 & 1.6 & - & - \\
\hline \multicolumn{5}{|l|}{ Plan type } \\
\hline Comprehensive & 297 & 31.2 & - & - \\
\hline Health maintenance organization & 105 & 11.0 & 69 & 12.2 \\
\hline Preferred provider organization & 493 & 51.7 & 405 & 71.7 \\
\hline Other & 44 & 4.6 & 75 & 13.3 \\
\hline Missing/unknown & 14 & 1.5 & 16 & 2.9 \\
\hline \multicolumn{5}{|l|}{ Data type } \\
\hline Commercial & 307 & 32.2 & 506 & 89.5 \\
\hline Medicare & 646 & 67.8 & 45 & 8.0 \\
\hline Other (Medicaid, missing, prescription only) & - & - & 14 & 2.5 \\
\hline \multicolumn{5}{|l|}{ Klabunde comorbidity score } \\
\hline 0 & 540 & 56.7 & 355 & 62.8 \\
\hline 1 & 222 & 23.3 & 114 & 20.2 \\
\hline $2+$ & 191 & 30.0 & 96 & 17.0 \\
\hline
\end{tabular}

external beam radiotherapy to relieve skeletal symptoms, new symptomatic pathologic vertebral or pathological bone fractures, spinal cord compression, and tumor-related orthopedic surgical intervention. ${ }^{3,16,17}$

Despite many therapeutic options available for treatment of $\mathrm{MCRPC}$, limited information is available around treatment patterns and their associated costs and health care resource utilization in a real-world setting. The purpose of this study was to describe the overall treatment patterns and health care costs in MCRPC patients with bone metastases who are treated with at least 1 agent approved by the U.S. Food and Drug Administration (FDA) for this indication.

\section{Methods}

\section{Study Design}

This was a retrospective, observational cohort study using administrative claims data from 2 large commercial and Medicare-insured U.S. integrated claims databases, Truven Health MarketScan and IMS LifeLink PharMetrics Plus. The MarketScan database contains inpatient and outpatient medical and prescription claims of employer and health plans. Enrollees included in the database are employees, their spouses, and dependents who are covered by employer-sponsored private health insurance. The PharMetrics database is composed of adjudicated claims that are representative of the national, commercially insured population in terms of age and gender for individuals aged 65 years and under. The majority of the MarketScan sample was covered under Medicare (67.8\%) along with commercial, whereas the PharMetrics sample was almost entirely commercial (89.5\%), with some Medicaid and no Medicare (Table 1)

\section{Patient Population}

Patients from MarketScan and PharMetrics databases were identified as eligible if they were a male with at least 1 primary or secondary diagnosis of PC as determined by the International Classification of Diseases, Ninth Revision, Clinical Modification (ICD-9-CM) for PC (ICD-9-CM code 185.xx) and had a claim for at least 1 FDA-approved treatment for mCRPC during the index period of June 1, 2013, to September 30, 2014. 
FDA-approved treatments included radium-223, sipuleucel-T, cabazitaxel, docetaxel, abiraterone, enzalutamide, mitoxantrone, or other chemotherapy, defined as any oral and injectable drugs indicated for mCRPC. Other chemotherapy agents included but were not limited to doxorubicin, etoposide, and paclitaxel. Adult patients who met diagnosis and treatment requirements must have been continuously active in the database for $>6$ months pretreatment (baseline period) and $\geq 6$ months post-treatment (follow-up period) from the index date. The date of the first claim for an FDA-approved treatment was defined as the index date. Patients must also have had a primary or secondary diagnosis indicating bone metastases identified by ICD-9-CM code 198.5 or any bone antiresorptive therapy to prevent or delay disease-associated skeletal-related events-including denosumab, zoledronic acid, and pamidronate-or palliative radiation therapy (strontium, samarium, and all other radiation). Claims were used to identify prescription treatments, and Current Procedural Terminology codes for radiation were used pre-index and during follow-up.

Patients meeting any of the following criteria were excluded: previous treatment with radium-223, sipuleucel-T, cabazitaxel, docetaxel, abiraterone, enzalutamide, or mitoxantrone ordiagnosis of cancer other than PC (ICD-9-CM codes 140.xx-171.xx, 174.xx184.xx, 186.xx-195.xx, 200.xx-209.3x, and 230.xx-239.xx) during the baseline period.

A subset of patients who developed new bone metastases before the study period was assessed. Inclusion in the subset required patients to be continuously eligible for at least 12 months before the treatment index date to establish a bone metastases index date. In addition, patients were required to have continuous database activity for 6 months before the bone metastases index date. Patients with a claim for bone metastases in the 6 months before the bone metastases index date were excluded.

\section{Outcomes}

Outcomes that were evaluated included treatment patterns, health care resource utilization (HCRU), health care costs, and mortality rates. Health care costs and resource utilization were evaluated within the 6-month follow-up period while treatment patterns and mortality were evaluated over a variable follow-up period based on patient data through the end of continuous enrollment or available data (March 31, 2015), whichever occurred first.

Treatment patterns included bone antiresorptive therapy (denosumab, zoledronic acid, pamidronate) or palliative radiation (strontium, samarium, other radiation); nonmutually exclusive distribution of treatment of PC by specific agents within each line of therapy; and duration of treatment for each PC treatment type over the variable follow-up period. First-line treatment was defined as any treatment given to a patient during the first 28 days ( 1 cycle) after bone metastases diagnosis. If a secondary treatment agent was given within 28 days of initiation of first-line treatment, this agent was defined as given in combination with the first agent in first line. If the secondary agent was given after 28 days, it was defined as given as second line in combination with the first agent. Drugs given for 1 or more cycles were considered within the same line of treatment. The end of first-line treatment was defined as the earliest observance of addition or substitution of any FDA-approved treatment or a gap of 90 days or more in therapy. Treatment lines followed the same logic as outlined for first-line treatment.

HCRU was reported per patient per month (PPPM) by setting of care including inpatient, outpatient, emergency department (ED), office visit, and pharmacy. Length of stay was reported per hospitalization. Health care costs were reported as medical costs, pharmacy costs, and total costs (medical plus pharmacy). Medical costs included office visits, outpatient visits, ED visits, inpatient stays, and medications provided under the medical benefit (e.g., radium-223, sipuleucel-T, and injectable chemotherapy). Pharmacy costs accounted for all costs for medications provided under the pharmacy benefit (e.g., oral chemotherapy). Costs were unadjusted and reported as direct costs to the payer (i.e., did not include patient cost share) from the study period of each database. Mortality was also assessed within the PharMetrics population.

The subset population of patients with newly diagnosed bone metastases was characterized by the first-line therapy option. Median time to therapy in this newly diagnosed bone metastases subset population was estimated based on the difference between the date of bone metastases diagnosis and the treatment index date.

\section{Statistical Analyses}

Standard descriptive analyses were used to describe baseline characteristics and outcomes associated with the management of PC patients with bone metastases. Continuous variables are represented by mean \pm standard deviation (SD) whereas categorical variables are represented by number and percentages. For specific outcomes (e.g., costs) where the distribution was skewed, data are presented by specific quartile or percentile information. The Wilcoxon signed-rank test for paired comparisons, which uses nonparametric data, was used to test for differences between pre- and post-index periods. Mortality data were only available within the PharMetrics database and were assessed as time to death via standard time-to-event calculations used with the Kaplan-Meier estimated survival function. Mortality rates were assessed using a landmark analysis of 6 months. Patients who died or were censored before 6 months were not included in this analysis.

\section{Results}

\section{Study Population}

Within databases, 418,355 males with at least 1 medical claim with a diagnosis of PC were identified in MarketScan and 


\section{FIGURE 1 Mean PPPM Health Care Resource Utilization}

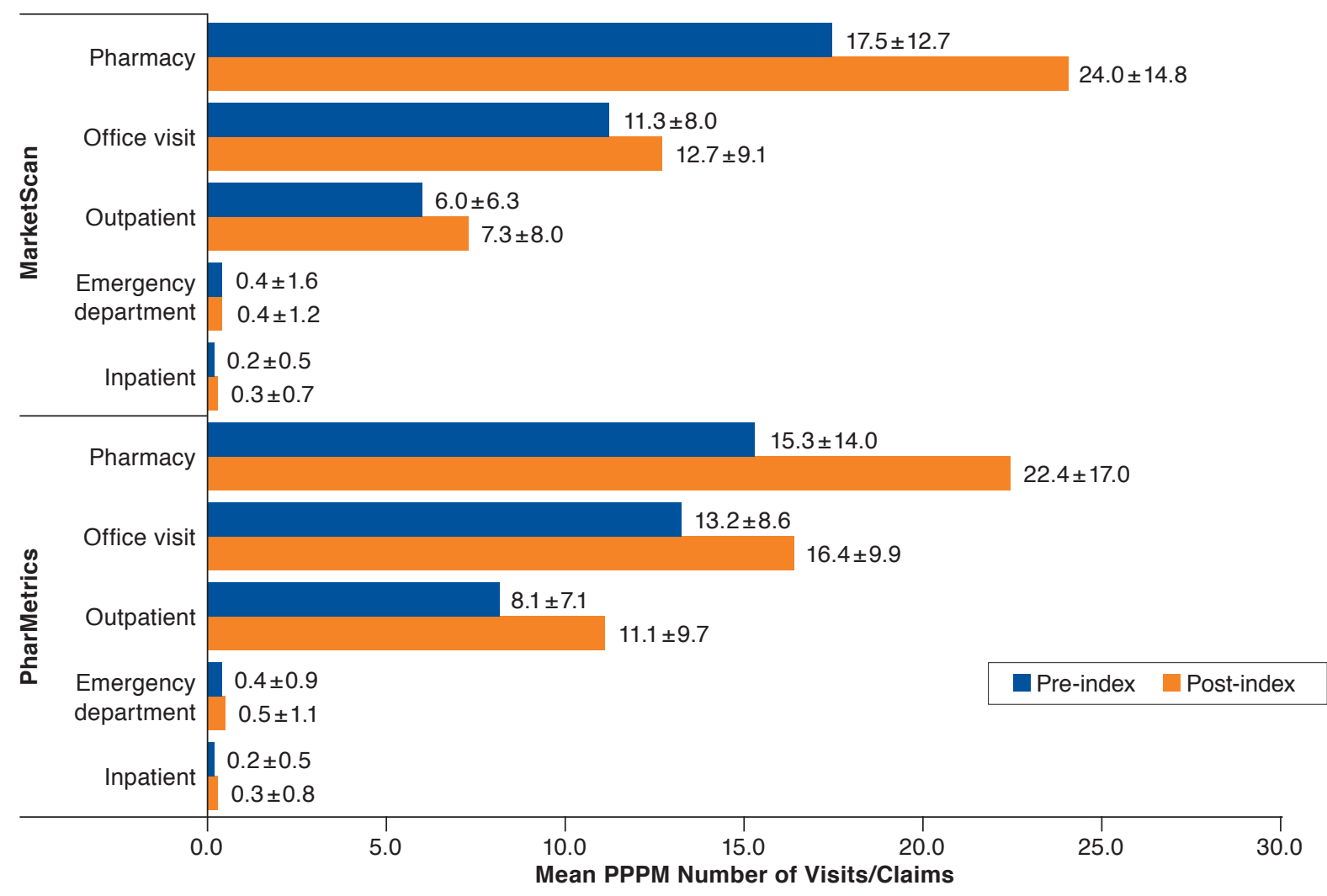

Note: For MarketScan: $P<0.05$ for all visits and claims except for $E D$ visits $(P=0.213)$. For PharMetrics: $P<0.05$ for all visits and claims except for ED visits ( $P=0.065)$. $E D=$ emergency department; $P P P M=$ per patient per month.

197,765 in PharMetrics. Among those, patients who received an FDA-approved treatment for $\mathrm{mCRPC}$ and had evidence of bone metastases during the pre-index period were identified: 2,188 patients in MarketScan and 1,273 in PharMetrics. After applying all exclusion criteria, 953 patients were selected from MarketScan and 565 from PharMetrics.

\section{Baseline Characteristics}

The age of the majority of patients in both datasets was at least 55 years (94.6\% in MarketScan and 90.6\% in PharMetrics). Mean ages were 71 years $( \pm 10.7)$ in MarketScan and 66 years $( \pm 9.3)$ in PharMetrics. In both datasets, few patients resided in the West (14.8\% and $7.8 \%$, respectively), and distribution in the Northeast, North Central/Midwest, and South ranged from $24.2 \%$ to $34.5 \%$. The majority of patients used preferred provider organization plans (51.7\% and $71.7 \%$ ). Disease burden in MarketScan and PharMetrics patients at baseline period were grouped by Klabunde scores of 0 (56.7\% and 62.8\%), 1 (23.3\% and $20.2 \%$ ), and 2 or higher (20.0\% and $17.0 \%)$.
The median follow-up time was 18 months (interquartile range $[\mathrm{IQR}]=14-23$ ) for MarketScan patients and 14 months (11-18) for PharMetrics patients. Before mCRPC treatment initiation, patients received palliative radiation therapy and bone antiresorptive therapy. For MarketScan and PharMetrics, respectively, $14.0 \%$ and $18.2 \%$ of patients received radiation therapy; $36.1 \%$ and $40.0 \%$ received denosumab; $16.5 \%$ and $16.8 \%$ received zoledronic acid; and $0.2 \%$ and $0.8 \%$ received pamidronate. Use of bone antiresorptive therapy increased in the follow-up period in comparison to baseline for all agents except pamidronate (e.g., denosumab increased to $44.8 \%$ in MarketScan and $47.4 \%$ in PharMetrics).

\section{Treatment Patterns}

Abiraterone was used in a large proportion of patients across all lines of therapy, followed by enzalutamide and docetaxel (Appendix A). The most commonly used treatment in firstline therapy in both databases was abiraterone (49.6\% in MarketScan and $42.5 \%$ in PharMetrics). Other chemotherapy (e.g., doxorubicin, etoposide, and paclitaxel) was the second 


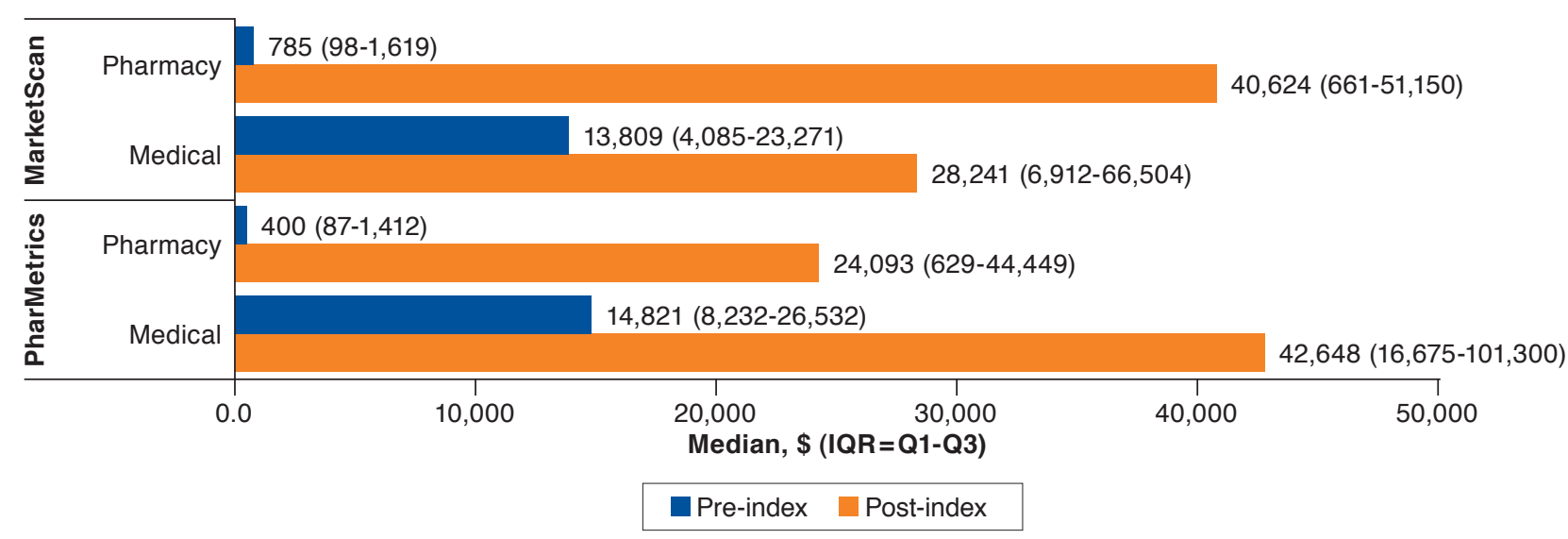

$I Q R=$ interquartile range; $Q 1-Q 3=$ quarter 1 to quarter 3.

most common choice used as first-line therapy for the MarketScan cohort (23.4\%), while docetaxel was second most for the PharMetrics cohort (26.9\%). Of patients who moved on to second-line therapy, the most common therapy was other chemotherapy (56.6\% and 62.1\%) followed by abiraterone (37.2\% and $37.7 \%)$, enzalutamide (20.5\% and $14.9 \%$ ), and docetaxel (14.6\% and $21.8 \%$ ). Of patients who moved to thirdline therapy, the most common therapy was abiraterone $33.7 \%$ and $33.5 \%$ ). The most common fourth-line chemotherapies were other chemotherapy agents (41.7\% and 38.1\%).

Among patients in the MarketScan database, median duration of treatment across any line of therapy was longest with other chemotherapy (9.2 months; IQR=4.9-13.4) followed by abiraterone (7.9 months; IQR =4.0-12.6), enzalutamide (5.0 months; IQR =2.6-8.5), and docetaxel (4.2 months; $\mathrm{IQR}=2.6-5.6)$. Among patients in the PharMetrics database, median treatment duration across any line of therapy was longest with abiraterone (7.8 months; IQR =3.9-11.9), followed by other chemotherapy (7.0 months; IQR=4.0-12.6), enzalutamide (4.6 months; IQR=2.1-9.1), and docetaxel (4.2 months; $\mathrm{IQR}=$ 2.8-5.6; Appendix A).

\section{Health Care Utilization and Costs Related to Prostate Cancer with Bone Metastases}

In both databases, mean PPPM HCRU increased from the baseline period to the follow-up period, as shown in Figure 1. In the MarketScan database, 283 (29.7\%) patients had an inpatient hospitalization within 6 months post-index versus $140(14.7 \%)$ patients at 6 months pre-index. This increase was consistent with that of the PharMetrics database, in which 167 (29.6\%) patients had an inpatient hospitalization during the 6-month follow-up period versus 105 (18.6\%) patients at
6 months pre-index. The mean (SD) length of stay increased from $4.4( \pm 3.6)$ to $4.7( \pm 4.2)$ days in MarketScan and $5.4( \pm 6.1)$ to 6.7 ( \pm 11.3$)$ days in PharMetrics.

Overall, pharmacy and medical costs substantially increased from the pre- to post-index periods (Figure 2). Median pharmacy costs increased from $\$ 785$ (IQR $=\$ 98$ $\$ 1,619)$ to $\$ 40,624(\mathrm{IQR}=\$ 661-\$ 51,150)$ for MarketScan and $\$ 400(\mathrm{IQR}=\$ 87-\$ 1,412)$ to $\$ 24,093(\mathrm{IQR}=\$ 629-\$ 44,449)$ for PharMetrics. Whereas median medical costs increased from $\$ 13,809$ (IQR $=\$ 4,085-\$ 23,271)$ to $\$ 28,241 \quad(\mathrm{IQR}=\$ 6,912$ $\$ 66,504)$ for MarketScan and $\$ 14,821(\mathrm{IQR}=\$ 8,232-\$ 26,532)$ to $\$ 42,648$ (IQR $=\$ 16,675-\$ 101,300)$ for PharMetrics. Median PPPM costs increased from baseline to post-index periods in both datasets substantially, by approximately $\$ 8,900$. MarketScan data showed that patients had a median treatment PPPM cost of $\$ 11,598(\mathrm{IQR}=\$ 6,840-\$ 16,627)$ post-index versus $\$ 2,643(\mathrm{IQR}=\$ 850-\$ 4,357)$ at baseline (Figure 3). PharMetrics data showed a median treatment PPPM cost of $\$ 11,601$ (IQR $=\$ 8,577-\$ 18,362$ ) post-index in comparison to $\$ 2,742$ (IQR $=\$ 1,484-\$ 4,730)$ at baseline. When median PPPM cost was evaluated for the entire variable follow-up period through the end of continuous enrollment or available data (March 31, 2015), costs were $\$ 10,916 \quad(\mathrm{IQR}=\$ 5,334$ $\$ 13,457)$ for MarketScan and $\$ 10,292(\mathrm{IQR}=\$ 7,245-\$ 14,699)$ for PharMetrics.

\section{New Bone Metastases}

For patients in the MarketScan database who developed new bone metastases, about half (345) were treated with abiraterone (Appendix B). Nearly the remainder of patients was treated with enzalutamide (18\%), docetaxel (16\%), and sipuleucel-T (14\%). Mean (SD) duration of therapy for abiraterone was 193 


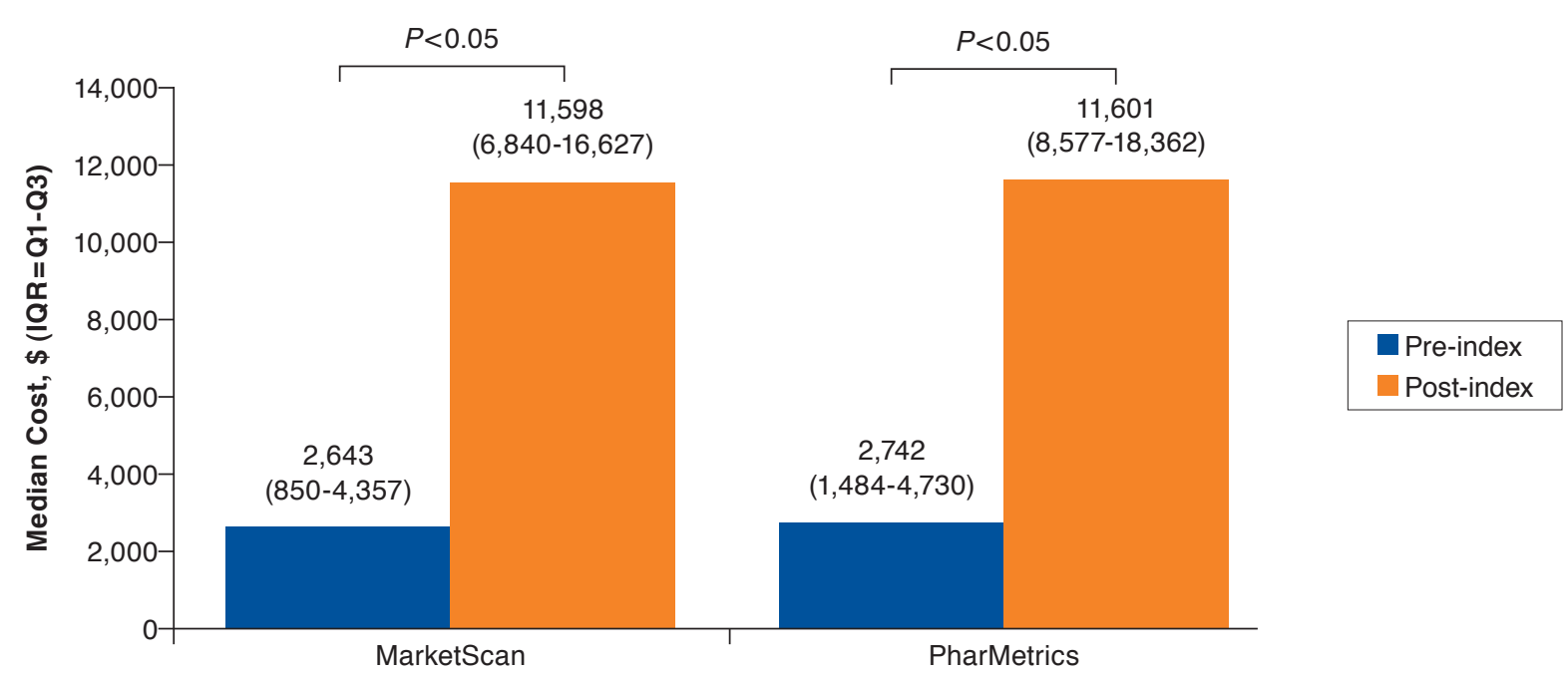

Note: Costs are shown in 2015 U.S. dollars.

$I Q R=$ interquartile range; $P P P M=$ per patient per month; $Q 1-Q 3=$ quarter 1 to quarter 3 .

days (131) in MarketScan and 150 days (118) in PharMetrics. Mean duration of therapy for enzalutamide was 211 days (122) in MarketScan and 200 days (98) in PharMetrics. The mean duration of treatment with any therapy averaged 184 days (132) for MarketScan and 134 days (115) for PharMetrics.

\section{Mortality}

Median OS was not captured because of the observation period. At 810 days (2.2 years) after initial treatment, it was estimated via Kaplan-Meier curve that $64.6 \%$ of PharMetrics patients were still alive (Figure 4). These data were not available for the MarketScan database.

\section{Discussion}

\section{Treatment Patterns}

Treatment patterns identified were comparable to the most recent NCCN and AUA guidelines for PC even though our patients were identified between June 2013 and September 2014. The NCCN Guidelines distinguish therapy based on location (visceral vs. nonvisceral) and symptomatic nature of metastases. Abiraterone and enzalutamide are recommended in visceral and nonvisceral CRPC patients, while docetaxel is preferred only in patients with visceral metastases. Cabazitaxel is used as a second-line option for both visceral and nonvisceral CRPC patients. For patients with asymptomatic or minimally symptomatic mCRPC with bone metastases, NCCN Guidelines recommend sipuleucel-T. For symptomatic CRPC patients, radium-223 is recommended. ${ }^{3}$ AUA guidelines align with NCCN Guidelines on these recommendations ${ }^{16}$ Results across both databases indicated abiraterone was the most commonly received agent in all lines of therapy, followed by enzalutamide and docetaxel. This is consistent with findings by Flaig et al. (2016) who demonstrated that abiraterone and enzalutamide were predominantly used as first and second lines of treatment. ${ }^{18}$ Abiraterone was approved 1 year before enzalutamide, which could explain the higher utilization. In patients receiving bone antiresorptive therapies or radiation, denosumab was the most common, followed by radiation and zoledronic acid.

A previous 2014 treatment utilization study evaluated the Premier Perspective Database-the largest U.S. inpatient drug utilization database for PC patients with and without bone metastases - to characterize therapy trends between January 2006 and December 2010. The results (not mutually exclusive) found that patients without bone metastases commonly used radiation (48.8\%), surgery (31.9\%), secondary hormone therapy (19.3\%), and primary hormone therapy (16.0\%). In patients with bone metastases, the trend was secondary hormone therapy (46.4\%), primary hormone therapy (44.3\%), chemotherapy (27.9\%), and radiation (24.0\%). ${ }^{15}$

In contrast, our results indicate that a higher percentage of patients use chemotherapy versus radiation, regardless of the presence of bone metastases. One reason for the use of chemotherapy in the nonmetastatic setting may be that physicians are aggressively treating patients for bone metastases without an official confirmatory test. The majority of hospitals participating in the Premier Perspective Database, however, are predominantly small- to medium-sized nonteaching facilities serving urban populations. CRPC patients are generally 


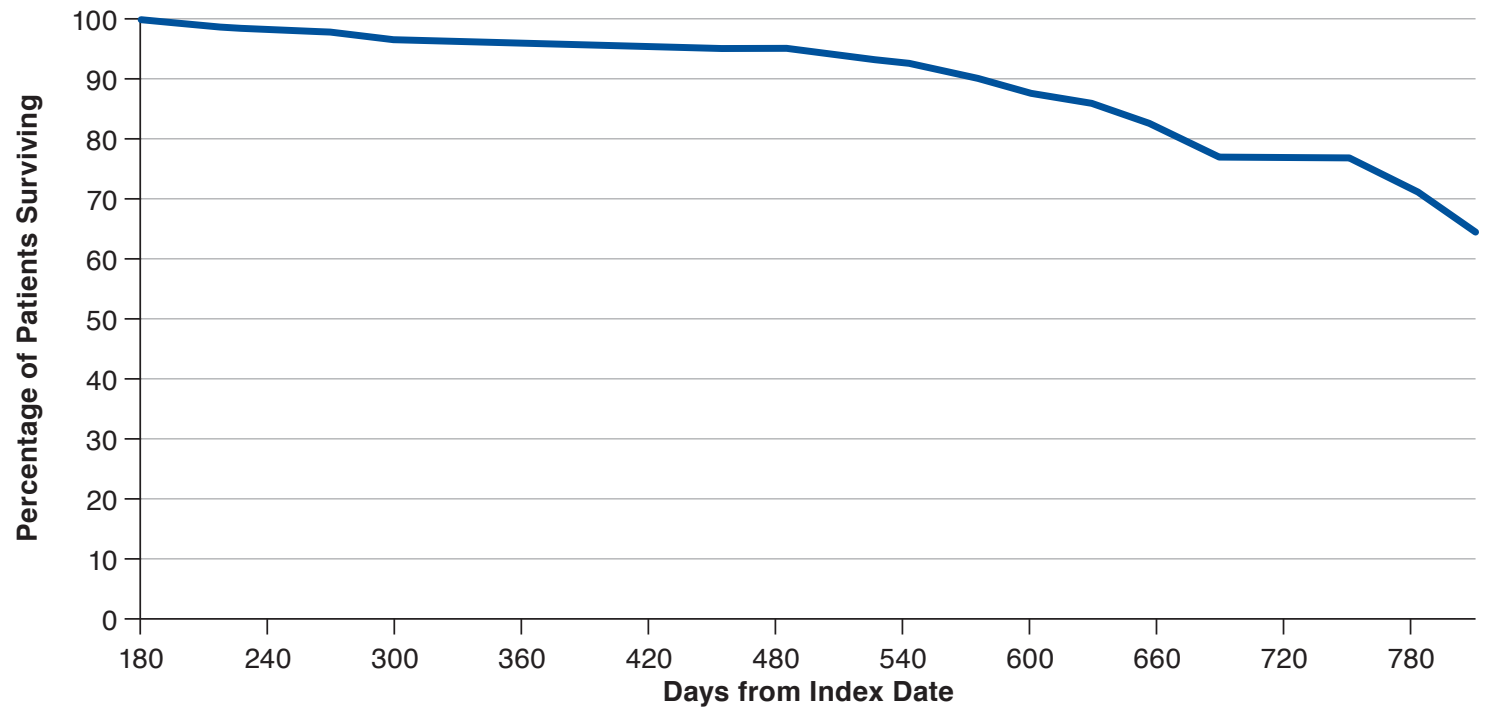

referred to teaching hospitals or cancer treatment centers. ${ }^{15}$ This information, in addition to the differing study periods, may account for some of the differences in treatment utilization findings.

Most treatment options fell short compared with their respective duration of treatment in pivotal trials and clinical studies with the exception of abiraterone. Abiraterone studies reported a median duration of therapy of 8.0 months in postchemotherapy patients and 13.6 months in prechemotherapy patients, which was close to our results of 7.9 for MarketScan and 7.8 for PharMetrics. Also consistent with our findings, a study evaluating postchemotherapy effectiveness of enzalutamide found that abiraterone median duration of therapy was 9.0 months and enzalutamide was 4.9 months. ${ }^{19}$ However, the median duration of therapy reported in the individual drug trials was most likely higher because of the controlled experimental design and population selection criteria compared with this real-world claims data study.

\section{Costs and Health Care Resource Utilization}

In this study, the number of patients with HCRU increased between baseline and follow-up periods for mCRPC. Baseline versus inpatient hospitalization rates after 6 months were $18.6 \%$ versus $29.6 \%$ for MarketScan and $14.7 \%$ versus $29.7 \%$ for PharMetrics. A 2014 retrospective analysis of the linked Surveillance, Epidemiology, and End Results (SEER) cancer registry and Medicare claims found that hospitalization rates among PC patients without distant metastases was $43.1 \%$, whereas hospitalization rates among PC patients with distant metastases were $60.9 \%$ at 1 year after diagnosis..$^{13}$ Our study only extended to 6 months, which may account for the differences.
Costs increased substantially between pre- and post-index periods among both databases. The median cost PPPM was $\$ 11,598$ (IQR $=\$ 6,840-\$ 16,627$ ) during 6 months post-index versus $\$ 2,643$ (IQR $=\$ 850-\$ 4,357$ ) during baseline for the MarketScan database. In PharMetrics, median PPPM was $\$ 11,601$ post-index (IQR $=\$ 8,577-\$ 18,362$ ) versus $\$ 2,742$ baseline (IQR $=\$ 1,484-\$ 4,730$ ). When considering all available data in the variable follow-up period, costs incurred in MarketScan and PharMetrics databases were $\$ 10,916 \quad(\mathrm{IQR}=\$ 5,334$ $\$ 13,457)$ and $\$ 10,292$ (IQR $=\$ 7,245-\$ 14,699)$, respectively. The difference in costs between 6-month post-index and post-variable follow-up was mostly because of a longer time frame across all patients, lowering the overall PPPM average. Of note, PharMetrics data contain only commercial claims and do not include Medicare claims, contributing to the younger demographic compared with MarketScan. Despite the younger demographic of PharMetrics, PPPM costs were similar between both datasets, although MarketScan may be more representative of the MCRPC population.

The median medical cost per patient was $\$ 13,809$ (IQR $=\$ 4,085-\$ 23,271)$ for the baseline period versus $\$ 28,241$ (IQR $=\$ 6,912-\$ 66,504)$ for the post-index period in the MarketScan database and $\$ 14,821$ (IQR $=\$ 8,232-\$ 26,532$ ) for the baseline period versus $\$ 42,648$ (IQR $=\$ 16,675-\$ 101,300$ ) for the post-index period in the PharMetrics database.

The increase in post-index medical costs may be attributed to increased office visits, outpatient visits, and length of hospital stays during the post-index period. Proportionally, the largest increase in costs overall was pharmacy costs. Perpatient pharmacy benefit costs totaled $\$ 785(\mathrm{IQR}=\$ 98-\$ 1,619)$ 
for the baseline period versus $\$ 40,624$ (IQR $=\$ 661-\$ 51,150$ ) for the post-index period in the MarketScan database and $\$ 400$ (IQR $=\$ 87-\$ 1,412$ ) for the baseline period versus $\$ 24,093$ (IQR $=\$ 629-\$ 44,449$ ) for the post-index period in the PharMetrics database. Post-index costs varied between groups because of age differences in the patient populations. In the MarketScan database, the majority of patients were aged over 65 years, which is associated with different utilization of medications under the medical versus pharmacy benefit compared with the utilization of younger patients in the PharMetrics database. The high rates of HCRU in the baseline period were because of the costs associated with the workup of mCRPC. A claims database found similar results among mCRPC patients, with costs rising before diagnosis, peaking during the month when metastases were diagnosed, and remaining significantly higher thereafter compared with a control group of PC patients who did not develop metastases. ${ }^{20}$ A previous study showed mean pharmacy costs for PC patients before bone metastases were $\$ 551$ compared with after metastases at $\$ 2,017 .{ }^{15} \mathrm{~A}$ rationale for the steep increase could be attributed to a variety of reasons. A major contributing factor could be because of initiation and high utilization of oral medications for hormonal therapy. Another contributor to costs is medication treatment of bone metastases. Findings suggest that more patients were being treated for bone metastases in the post-index period versus the baseline period. Medication treatment costs of these bone metastases can be substantial, with the highest costs totaling over $\$ 1,000$ per injection. ${ }^{21}$

\section{Disease-Related Outcomes}

Data indicated that $64 \%$ of patients with PC and bone metastases were alive 810 days (2.2 years) after initial treatment. SEER reported that the 5 -year survival rate in distant PC is $29.3 \%{ }^{22}$ If this study were to extend for a total of a 5-year period, we would anticipate similar findings to that of SEER data.

\section{Limitations}

There are inherent limitations that exist when using claims databases, since claims data are not collected for purposes of research and are susceptible to coding errors. Analysis was also limited in identification of the patient population by proxy of available data and short follow-up time period of 6 months. An algorithm for identifying CRPC patients has not been validated in the literature. However, there is a strong likelihood that CRPC patients were appropriately captured based on the disease-specific agents prescribed. ICD-9-CM codes, specifically for bone metastases, are not entirely reliable targets for diagnosis dates. Patients may be diagnosed as metastatic by the physician, but a claim may not occur until the time of first treatment. As such, pre- and post-index periods may have been affected and occurred later than the official diagnosis.

Claims data also may lead to unanswered questions around the rationale for treatment discontinuation or changes in treatment regimen. Therefore, it is difficult to determine treatment failures versus patient unwillingness to continue because of side effects, tolerability, and other unaccounted factors. Since these were retrospective data, the picture of the current market in CRPC may not be complete because the drugs selected for this study are more recent market entrants. True market utilization may be different now in comparison to when the study was conducted, as it is continuously evolving and dynamic. Docetaxel may also be used in hormone-sensitive PC in addition to mCRPC, although, given this study's time period, most hormone-sensitive patients from these data would have predated the Chemohormonal Therapy in Metastatic HormoneSensitive Prostate Cancer (CHAARTED) and Systemic Therapy in Advancing or Metastatic Prostate Cancer: Evaluation of Drug Efficacy (STAMPEDE) trial publications, which supported docetaxel use in hormone-sensitive patients. At the time of this research, some treatment options were recently approved and may not reflect a complete view of market uptake and utilization.

\section{Conclusions}

Treatment patterns observed in this study aligned with currently available guideline recommendations. Abiraterone was the most frequently prescribed therapy across most lines of care, with enzalutamide and docetaxel also being commonly used. Median PPPM costs increased over the study period in both datasets, despite a younger demographic in the PharMetrics population. The total medical and pharmacy costs per patient increased substantially between baseline and post-index period, with pharmacy costs accounting for greater change. Additional studies from real-world settings, including Medicare populations, would provide additional perspectives on treatment utilization and potentially explore whether specific sequences of therapies can affect clinical outcomes.

\section{Authors}

LONNIE WEN, RPh, PhD; JIANYING YAO, MS; and ADRIANA VALDERRAMA, PhD, MBA, Bayer HealthCare Pharmaceuticals, Whippany, New Jersey.

AUTHOR CORRESPONDENCE: Lonnie Wen, RPh, PhD, Bayer HealthCare Pharmaceuticals, 100 Bayer Blvd., Bldg. 200, 2nd Fl., Mailstop 2-14, PO Box 915, Whippany, NJ 07981-0915.

Tel.: 832.221.4095; E-mail: lonnie.wen@bayer.com. 


\section{DISCLOSURES}

Funding for this study was provided by Bayer HealthCare Pharmaceuticals. All authors were employees at Bayer HealthCare Pharmaceuticals at the time this study was conducted.

This study was presented as a poster at the 2017 American Society of Clinical Oncology (ASCO) Genitourinary Cancers Symposium; February 16-18, 2017; Orlando, FL.

\section{ACKNOWLEDGMENTS}

The authors acknowledge Victor Nguyen, PharmD; Melissa McCart, PharmD, MS; and Kylie Matthews of Xcenda, AmerisourceBergen, for editorial support.

\section{REFERENCES}

1. Noone AM, Howlader N, Krapcho M, et al., eds. SEER Cancer Statistics Review, 1975-2015. Updated September 10, 2018. National Cancer Institute Bethesda, MD. Available at: https://seer.cancer.gov/csr/1975_2015/. Accessed February 25, 2019

2. Amling CL, Blute ML, Bergstralh EJ, Seay TM, Slezak J, Zincke H Long-term hazard of progression after radical prostatectomy for clinically localized prostate cancer: continued risk of biochemical failure after 5 years. J Urol. 2000;164(1):101-05.

3. Mohler JL, Armstrong AJ, Bahnson RR, et al. Prostate cancer, version 1.2016. J Natl Compr Canc Netw. 2016;14(1):19-30.

4. Kirby M, Hirst C, Crawford ED. Characterising the castration-resistant prostate cancer population: a systematic review. Int J Clin Pract. 2011;65(11): 1180-92.

5. Hellerstedt BA, Pienta KJ. The current state of hormonal therapy for prostate cancer. CA Cancer J Clin. 2002;52(3):154-79.

6. Saad F, Hotte SJ. Guidelines for the management of castrate-resistant prostate cancer. Can Urol Assoc J. 2010;4(6):380-84.

7. Scher HI, Solo K, Valant J, Todd MB, Mehra M. Prevalence of prostate cancer clinical states and mortality in the United States: estimates using a dynamic progression model. PloS One. 2015;10(10):e0139440.

8. Tannock IF, de Wit R, Berry WR, et al. Docetaxel plus prednisone or mitoxantrone plus prednisone for advanced prostate cancer. N Engl J Med. 2004;351(15):1502-12

9. Petrylak DP, Tangen CM, Hussain MH, et al. Docetaxel and estramustine compared with mitoxantrone and prednisone for advanced refractory prostate cancer. N Engl J Med. 2004;351(15):1513-20.
10. de Bono JS, Logothetis CJ, Molina A, et al; COU-AA-301 Investigators. Abiraterone and increased survival in metastatic prostate cancer. N Engl J Med. 2011;364(21):1995-2005.

11. Jin JK, Dayyani F, Gallick GE. Steps in prostate cancer progression that lead to bone metastasis. Int J Cancer. 2011;128(11):2545-61.

12. Nørgaard M, Jensen A $\varnothing$, Jacobsen JB, Cetin K, Fryzek JP, Sørensen HT. Skeletal related events, bone metastasis and survival of prostate cancer: a population based cohort study in Denmark (1999 to 2007). J Urol. 2010;184(1):162-67.

13. Yong C, Onukwugha E, Mullins CD, Seal B, Hussain A. The use of health services among elderly patients with stage IV prostate cancer in the initial period following diagnosis. J Geriatr Oncol. 2014;5(3):290-308.

14. McDougall JA, Goulart BH, Sullivan SD, et al. Impact of skeletal related events on survival in Medicare patients with prostate cancer metastatic to bones. Poster presented at: ASCO Meeting; May 29-June 2, 2016; Chicago, IL. 15. Seal B, Sullivan SD, Ramsey SD, et al. Comparing hospital-based resource utilization and costs for prostate cancer patients with and without bone metastases. Appl Health Econ Health Policy. 2014;12(5):547-57.

16. Cookson MS, Roth BJ, Dahm P, et al. Castration-resistant prostate cancer: AUA guideline. J Urol. 2013;2(190):429-38.

17. Shore ND. Radium-223 dichloride for metastatic castration-resistant prostate cancer: the urologist's perspective. Urology. 2015;85(4):717-24.

18. Flaig TW, Potluri RC, Ng Y, Todd MB, Mehra M. Treatment evolution for metastatic castration-resistant prostate cancer with recent introduction of novel agents: retrospective analysis of real-world data. Cancer Med. 2016;5(2):182-91

19. Schrader AJ, Boegemann M, Ohlmann CH, et al. Enzalutamide in castration-resistant prostate cancer patients progressing after docetaxel and abiraterone. Eur Urol. 2014;65(1):30-36.

20. Li TT, Shore ND, Mehra M, et al. Impact of subsequent metastases on costs and medical resource use for prostate cancer patients initially diagnosed with localized disease. Cancer. 2017;123(18):3591-601.

21. Comer B. Xofigo approval keeps the ball rolling for Bayer Oncology. PharmExec.com. May 17, 2013. Available at: http://www.pharmexec.com/ xofigo-approval-keeps-ball-rolling-bayer-oncology. Accessed February 8, 2019.

22. American Cancer Society. Cancer facts \& figures 2017. Available at: https://www.cancer.org/content/dam/cancer-org/research/cancer-factsand-statistics/annual-cancer-facts-and-figures/2017/cancer-facts-and-figures-2017.pdf. Accessed February 15, 2019. 
APPENDIX A Line of Therapy and Median Duration Summary

\begin{tabular}{|c|c|c|c|c|c|c|c|c|c|c|}
\hline & \multicolumn{2}{|c|}{ First Line, \% } & \multicolumn{2}{|c|}{ Second Line, \% } & \multicolumn{2}{|c|}{ Third Line, \% } & \multicolumn{2}{|c|}{ Fourth Line, \% } & \multicolumn{2}{|c|}{$\begin{array}{l}\text { Median Duration, } \\
\text { Months (Q1-Q3) }\end{array}$} \\
\hline & MarketScan & PharMetrics & MarketScan & PharMetrics & MarketScan & PharMetrics & MarketScan & PharMetrics & MarketScan & PharMetrics \\
\hline Radium-223 & 2.1 & 2.7 & 1.6 & 2.3 & 2.2 & 2.5 & 1.6 & 1.9 & $3.7(2.0-5.6)$ & $4.0 \quad(1.9-5.9)$ \\
\hline Abiraterone & 49.6 & 42.5 & 37.2 & 37.7 & 33.7 & 33.5 & 26.3 & 26.4 & $7.9(4.0-12.6)$ & $7.8(3.9-11.9)$ \\
\hline Sipuleucel-T & 14.7 & 18.6 & 3.0 & 3.0 & 0.7 & 1.2 & 0.7 & 0.4 & $1.4 \quad(1.4-1.6)$ & $1.4 \quad(1.4-1.6)$ \\
\hline Docetaxel & 16.4 & 26.9 & 14.6 & 21.8 & 12.2 & 17.3 & 9.9 & 12.9 & $4.2(2.6-5.6)$ & $4.2 \quad(2.8-5.6)$ \\
\hline Cabazitaxel & 0.8 & 1.1 & 1.3 & 1.6 & 1.5 & 1.8 & 2.1 & 3.2 & $2.1 \quad(1.4-4.0)$ & $2.1 \quad(1.1-3.5)$ \\
\hline Enzalutamide & 18.4 & 10.3 & 20.5 & 14.9 & 21.2 & 13.6 & 19.8 & 13.8 & $5.0(2.6-8.5)$ & $4.6 \quad(2.1-9.1)$ \\
\hline Mitoxantrone & 0.1 & 0.2 & 0.2 & 0.4 & 0.2 & 0.2 & 0.1 & 0.2 & $2.4 \quad(2.1-6.4)$ & $1.4 \quad(0.7-2.1)$ \\
\hline Antiandrogen & 6.2 & 7.8 & 7.8 & 8.0 & 5.8 & 6.0 & 4.3 & 5.0 & $3.2 \quad(1.9-7.4)$ & $4.0 \quad(2.1-7.5)$ \\
\hline $\begin{array}{l}\text { Other } \\
\text { chemotherapy }\end{array}$ & 23.4 & 25.5 & 56.6 & 62.1 & 28.3 & 31.5 & 41.7 & 38.1 & $9.2(4.9-13.4)$ & $7.0(4.0-12.6)$ \\
\hline
\end{tabular}

Note: These results are not mutually exclusive. Not all patients moved through all 4 lines of therapy.

Q1-Q3 = quarter 1 to quarter 3 .

APPENDIX B Days to Treatment in Newly Diagnosed Bone Metastases Population

\begin{tabular}{|c|c|c|c|c|c|c|}
\hline \multirow[b]{3}{*}{ Abiraterone } & \multicolumn{3}{|c|}{ MarketScan } & \multicolumn{3}{|c|}{ PharMetrics } \\
\hline & \multirow{2}{*}{$\frac{\mathrm{n}}{345}$} & \multirow{2}{*}{$\begin{array}{c}\% \\
49.6\end{array}$} & Mean (SD), days & \multirow{2}{*}{$\frac{n}{120}$} & \multirow{2}{*}{$\frac{\%}{39.0}$} & Mean (SD), days \\
\hline & & & $193 \quad(131)$ & & & $150 \quad(118)$ \\
\hline Cabazitaxel & 6 & 0.9 & $235 \quad(170)$ & 1 & 0.3 & $318 \quad(\mathrm{NA})$ \\
\hline Docetaxel & 113 & 16.2 & $157 \quad(132)$ & 93 & 30.2 & $100 \quad(105)$ \\
\hline Enzalutamide & 125 & 18.0 & $211 \quad(122)$ & 27 & 8.8 & 200 \\
\hline Mitoxantrone & 1 & 0.1 & $316 \quad(\mathrm{NA})$ & 0 & 0.0 & NA \\
\hline Radium-223 & 7 & 1.0 & $226 \quad(107)$ & 3 & 1.0 & $155 \quad(136)$ \\
\hline Sipuleucel-T & 99 & 14.2 & $139 \quad(130)$ & 64 & 20.8 & $121 \quad(111)$ \\
\hline $\begin{array}{l}\text { Total new bone metastases } \\
\text { patients }\end{array}$ & 696 & 100.0 & $184 \quad(132)$ & 308 & 100.0 & $134 \quad(115)$ \\
\hline
\end{tabular}

NA = not available; $21-Q 3=$ quarter 1 to quarter $3 ; S D=$ standard deviation. 


\section{Supplement}

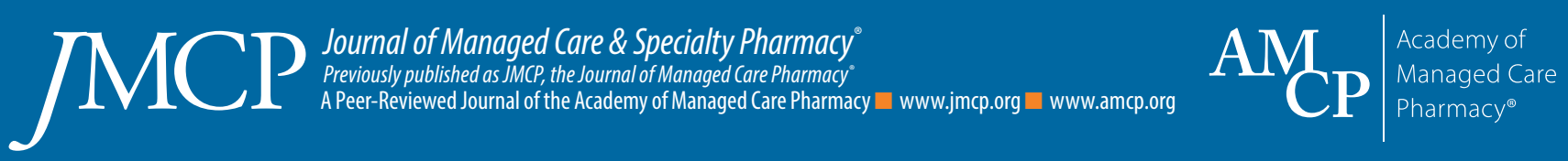

